\title{
Isolated medial patellofemoral ligament reconstruction significantly improved quality of life in patients with recurrent patella dislocation: a response to Hiemstra et al.'s letter to the editor
}

Theodoros Bouras $^{1}$, Jan Herman Kuiper ${ }^{1,2}$ and Andrew Barnett ${ }^{1}$

1. Sports Knee Department, The Robert Jones and Agnes Hunt Orthopaedic Hospital, Oswestry, UK

2. School of Pharmacy and Bioengineering, Keele University, Keele, UK

Published as: Bouras T, Kuiper JH, Barnett A. Isolated medial patellofemoral ligament reconstruction significantly improved quality of life in patients with recurrent patella dislocation: a response to Hiemstra et al.'s letter to the editor. Knee Surg Sports Traumatol Arthrosc. 2019 Nov;27(11):37353737. doi: 10.1007/s00167-019-05690-1.

\section{LETTER TO THE EDITOR}

This reply refers to the article available at https://doi.org/10.1007/s00167-019-05618-9.

Dear Professor Karlsson,

Thank you very much for your recent correspondence and in particular the letter from Hiemstra et al. [5]. We thank them for their careful reading of our publication and gladly use this opportunity to address the issues they raise.

We acknowledge that they have undertaken a significant amount of research work in this field, having first published the Banff Patellar Instability Instrument (BPII) in 2013. It was certainly not our intention to undermine the BPII in any way. Our aim was to report general health related and not condition specific Quality of Life (QoL) outcome for our patients, in addition to health-economic outcomes, using the EQ-5D questionnaire. We apologise if this was not easily understood when reading our paper.

The statement "no study has specifically looked at quality of life (QoL)" is indeed incorrect, as pointed out by Hiemstra et al. [5]. However, our full statement in the paper reads "no study has specifically looked at quality of life (QoL) and (emphasis added) health-economic outcomes, in particular the health state preference value (utility), following surgery for patellofemoral instability.", and to our knowledge this is a correct statement.

Hiemstra et al. question the suitability of the EQ-5D-3L as our instrument to measure healtheconomic and health-related QoL outcomes in this specific patient population [5]. The EQ-5D is a Generic Preference Based M easure (GPBM ). GPBM s can incorporate the impact of treatment or ill health on a multidimensional scale and are useful in comparing between interventions and across conditions [8]. However, GPM Bs have been criticised for being insensitive or failing to capture 
important aspects of health, which also seems the criticism raised by Hiemstra et al. [4, 8]. Several Condition-Specific Preference Based M easures (CSPBM s) have therefore been developed for a number of diseases, albeit only one for a musculoskeletal condition (arthritis) [4, 13]. The outcomes quoted by Hiemstra et al. are not preference-based outcomes and thus less suitable for health economic evaluations. The arthritis-specific preference based measure was not better than the EQ5D [13]. This should be no surprise, since comparisons across diseases and conditions show that musculoskeletal conditions have among the largest impact on the EQ-5D [12, 14]. This is also depicted in a recent systematic review that Hiemstra et al. reference to point low responsiveness for lower limb reconstruction surgery. However, they neglect to report that EQ-5D was responsive for elective upper and lower limb surgery, including primary Total Knee Replacement (TKR) and primary Total Hip Replacement (THR) [10]. EQ-5D-3L has successfully been used to measure quality of life in soft tissue reconstruction procedures in the past $[3,15]$. A most recent cohort study compared the internal and external responsiveness of the EQ-5D-3L in the setting of knee arthroscopy, with two other Patient Reported Outcome scores (PROs), concluding in recommending either of these PROs for soft tissue procedures [9].

In our study the median baseline utility score was 0.69 as measured by the EQ-5D index. This is in line with scores for chronic musculoskeletal conditions, suggesting the EQ-5D is sufficiently sensitive to capture the impact of patellar instability on the health state of our patients. The rise in median utility score from 0.69 to 1.0 after the operation suggests it also captures well the effect of the operation. As Hiemstra et al. point out, our results had a strong ceiling effect, with the majority of our patients reporting a utility value of 1 ("perfect health") after the operation. This was no surprise to us, since the large ceiling effect of the EQ-5D has been well documented [12, 14]. It simply means that the EQ-5D may not be fully able to distinguish between respondents whose health statuses are at the upper end of the scale, but that was never the aim of our study. Of course, this issue might be important if our aim had been to compare between treatments, for instance two types of surgery. However, in terms of health economics the relevant comparison would then be between the accrued benefit over time of the treatments, as measured in Quality Adjusted Life Years (QALYs), compared to the difference in costs. In other words, the durability of the outcomes comes in play, together with costs of further surgery, and in practice this is again well assessed using the EQ-5D [8].

Hiemstra et al. are also critical of the Kujala score, which they describe as "an anterior knee pain scale that does not measure patellofemoral instability, knee function or QoL" [5]. However, this description contradicts their account of the same score in their excellent paper from 2016 [6], where they explain it was developed to evaluate the subjective symptoms and functional limitations in patients with patellofemoral disorders and tested on four different patient cohorts, including anterior knee pain, patellar subluxation and patellar dislocation, and controls. Their paper also describes a moderately strong correlation between the Kujala score and two patellar instability specific scores, the BPII and the Norwich score, which they see as evidence that the three scores are measuring similar although not exactly the same constructs.

When we set up our prospective patellofemoral database in 2012, patellar instability specific scores were not available. At the time the Kujala score was the most frequently used outcome score for these disorders in the United Kingdom and hence we chose this as our measure of functional outcome pre and postoperatively. Hiemstra et al.'s results [6] suggest that this was indeed a 
reasonable choice, although we will certainly consider using a patellar instability specific score such as the BPII in the future.

Finally, the authors question our use of the multilevel (or mixed) model. We would like to point out that the mixed model is one of only two methods recommended by the European Medicines Agency (EMA) to handle missing data [2]. Rules of thumb are not necessarily the best guide to sample sizes: a widely respected textbook on mixed modeling uses 48 dataset with sample sizes ranging from 5 to 136 [11]. It is therefore always better to tailor the sample size to the study, which we did using our power analysis. Since we expected heteroskedasticity (see the above discussion on ceiling effects), we used a robust variance estimator in our analyses. These sandwich estimators are widely used in econometrics, where highly skewed distributions are the norm, and effectively deal with such issues [1]. Although we did not include it in the paper, we also performed the analysis of EQ-5D utility and VAS values using a mixed beta regression model, which fits the data better [7], but we found almost identical values in terms of means and differences between them. This small difference was also reported by Hunger et al., and therefore we refrained from reporting it [7]. Colinearity is only an issue when at least two covariates highly correlate, but it should come as no surprise this was not the case among our three covariates (age, gender and BM I).

\section{References}

1. Cameron AC, Trivedi PK (2005) M icroeconometrics: methods and applications. Cambridge University Press, Cambridge. ISBN 9780521848053

\section{European M edicine Agency (2010) Guideline in confirmatory clinical trials. CPMP/EWP/1776/99} Rev. 1

3. Fältström A, Hägglund M , Kvist J (2013) Patient-reported knee function, quality of life, and activity level after bilateral anterior cruciate ligament injuries. Am J Sports Med 41(12):2805-2813

4. Goodwin E, Green C (2016) A systematic review of the literature on the development of conditionspecific preference-based measures of health. Appl Health Econ Health Policy 14(2):161-183

5. Hiemstra LA, Kerslake S, Lafave M (2019) Letter to the editor. Knee Surg Sports Traumatol Arthrosc. https://doi.org/10.1007/s00167-019-05618-9

6. Hiemstra LA, Kerslake S, Lafave M, M ohtadi NG (2016) Concurrent validation of the banff patella instability instrument to the norwich patellar instability score and the kujala score in patients with patellofemoral instability. Orthop J Sports M ed 4(5):2325967116646085Central

7. Hunger M, Döring A, Holle R (2012) Longitudinal beta regression models for analyzing healthrelated quality of life scores over time. BM C M ed Res M ethodol 12(1):144Central

8. Longworth L, Yang Y, Young T, M ulhern B, Hernández Alava M, M ukuria C, Brazier J (2014) Use of generic and condition-specific measures of health-related quality of life in NICE decision-making: a systematic review, statistical modelling and survey. Health Technol Assess 18(9):1-224Central

9. Oak SR, Strnad GJ, Bena J, Farrow LD, Parker RD, Jones M H, Spindler KP (2016) Responsiveness comparison of the EQ-5D, PROM IS global health, and VR-12 questionnaires in knee arthroscopy. Orthop J Sports Med 4(12):2325967116674714Central 
10. Payakachat N, Ali M M , Tilford JM (2015) Can the EQ-5D detect meaningful change? A systematic review. PharmacoEconomics 33(11):1137-1154Central

11. Pinheiro JC, Bates DM (2000) Mixed-effects models in s and S-PLUS. Springer, New York. https://doi.org/10.1007/b98882

12. Saarni SI, Härkänen T, Sintonen H, Suvisaari J, Koskinen S, Aromaa A, Lönnqvist J (2006) The impact of 29 chronic conditions on health-related quality of life: a general population survey in Finland using 15D and EQ-5D. Qual Life Res 15(8):1403-1414

13. Versteegh M M, Leunis A, Uyl-de Groot CA, Stolk EA (2012) Condition-specific preference-based measures: benefit or burden? Value Health Reg Issues 15(3):504-513

14. Wu M, Brazier JE, Kearns B, Relton C, Smith C, Cooper L (2015) Examining the impact of longstanding health conditions on health-related quality of life using the EQ-5D in a general population sample. Eur J Health Econ 16(2):141-151

15. Zaffagnini S, Grassi A, Marcheggiani Muccioli GM , Luetzow WF, Vaccari V, Benzi A, M arcacci M (2014) Medial patellotibial ligament (MPTL) reconstruction for patellar instability. Knee Surg Sports Traumatol Arthrosc 22(10):2491-2498 